blue coloured ring in periphery of iris (11\%), dysmetria (9\%), gingivitis $(7 \%)$, intention tremor $(5 \%)$, decreased mental labial reflex (5\%), decreased Babinski reflex (5\%), ataxia (3\%) and decreased ankle jerk reflex (2\%). About 50\% of participants with clinical signs of mercury intoxication were found to have mercury levels in their blood and urine above WHO standards.

Discussion Our findings show higher levels of mercury in urine, blood and hair above recommended values which correspond to the neurological symptoms. There is a need for interventions on the reduction of the mercury exposure among workers.

\section{TRENDS AND PATTERNS OF FATAL OCCUPATIONAL INJURIES AND COMPENSATED OCCUPATIONAL DISEASES IN TAIWAN, 1998-2016}

Chung-Yen Chen*, Yawen Cheng. Institute of Health Policy and Health Management, National Taiwan University, Taipei, Taiwan

\subsection{6/oemed-2018-ICOHabstracts.431}

Introduction This study described the trends and compensation patterns of fatal occupational injuries and occupational diseases in Taiwan and examined the similarities and differences with that of selected countries.

Methods Numbers, occurrence rates and compensation benefits of fatal occupational injuries and occupational diseases in Taiwan were obtained from official statistics for the period from 1998 to 2016. Also obtained were official statistics on the numbers and major types of compensated occupational diseases from Japan, South Korea and selected European countries.

Results From 1998 to 2016, the coverage of the workers' compensation insurance program expanded from $79.6 \%$ to $86.7 \%$, and occupational fatality had declined substantially but was still higher as compared to other developed countries. Analyses of the levels of claimants' compensation benefits showed that the average amount of benefits for fatal cases had been reduced steadily. Despite of recent efforts by the government in improving the recognition of occupational disease, the compensation rates of occupational diseases remained low (8.18 per 100000 insured). Musculoskeletal disorders were the most dominant type of occupational disease in Taiwan, accounting for up to $66 \%$ in 2016 , following by respiratory diseases $(17 \%)$ and stress-related cerebrovascular and cardiovascular diseases (10\%).

Discussion The observed improvement in occupational fatality had been accompanied by a downward trend in average compensation benefits, suggesting a shift of occupational fatalities toward low-wage groups. On one hand, under-recognition and under-compensation of occupational diseases were severe, that may due to multiple institutional and administrative barriers as well as lower social awareness on the work-relatedness of disease causality. On the other hand, the high visibility of cerebrovascular and cardiovascular events suggested the social concerns over long working hours and heavy workloads might have played a significant role in the recognition of overworkrelated occupational diseases.

\section{9 LONGITUDINAL ASSOCIATIONS OF SHIFT WORK WITH DEPRESSIVE DISORDERS - A SYSTEMATIC REVIEW AND META-ANALYSIS}

Peter Angerer*, Renate Schmook, Jian Li. Institute for Occupational, Social, and Environmental Medicine, Medical Faculty, Heinrich-Heine-Universität Düsseldorf, Germany

\subsection{6/oemed-2018-ICOHabstracts.432}

Background Shift work, especially night shift work, causes disturbance of sleep, tiredness, and reduced well-being, as it interferes with the circadian chronobiological rhythm and social activities. Shift increases the risk of coronary heart disease and diabetes. Whether shift work increases the risk of depressive disorders is controversial. Whereas crossectional studies indicate an increased risk, longitudinal studies are inconclusive.

Methods Based on a systematic and extensive literature research in PubMed, Scopus, PsycINFO, PSYNDEX, and Medpilot, 5682 publications dealing with shift work and mental illness were identified until 2016 December. According to predefined selection criteria eleven high quality studies with longitudinal design reporting the relationships between shift work including night work and depressive disorders were analysed.

Results Three out of four studies, restricted in health care professions, predominantly nurses, found no significant relationships between shift work including night work and depressive disorders within period of two-year follow-up. Another five studies among employees working in sectors other than health care yielded indications for an increased risk, during period of up to ten years follow-up, however, a consistent pattern of longitudinal associations was not observed. Supplementary meta-analysis, including five studies, also indicated that shift work elevated the risk of depressive disorders by 42\% (95\% confidence interval 0.92-2.42). Adverse psychosocial work conditions may partly explain the associations.

Conclusions Even though the findings indicate an increased risk of depressive disorders by shift work or night work, at least outside the health care sector, the evidence is too weak to give general medical advice against shift work with respect to employees' mental health. Rather, it seems adequate to take an individualised approach with continuous support from occupational physicians and practical consideration on psychosocial stress factors that are linked to shift work. How these finding are incorporated in the German guidelines for shift work that are currently updated will be reported.

\section{EPIDEMIOLOLOGICAL PROFILE OF LABOR DISABILITY - IN PARATY}

${ }^{1}$ L Rozemberg, ${ }^{2} \mathrm{D}$ Rozemberg. ${ }^{1}$ Hospital Stela Maris, Caraguatatuba, Brazil; ${ }^{2}$ Ministry of Social Security, Paraty, Brazil

\subsection{6/oemed-2018-ICOHabstracts.433}

Introduction The causes of temporary incapacity, established through official expertise (Ministry of Welfare) in Paraty unit, Brazil, are evaluated by establishing the epidemiological profile aiming the proposal of preventive measures.

Methods Retrospective study of all skills that generated financial support for disability in the year 2016 in the city of 